\title{
Diffusion MRI: Mitigation of Magnetic Field Inhomogeneities
}

\begin{abstract}
P. Marcon $^{1}$, K. Bartusek ${ }^{2}$, Z. Dokoupil ${ }^{2}$, E. Gescheidtova ${ }^{1}$
${ }^{1}$ Department of Theoretical and Experimental Electrical Engineering, Faculty of Electrical Engineering and Communication, Brno University of Technology, Kolejni, 2906/4., 612 00, Brno, Czech Republic, marcon@feec.vutbr.cz

${ }^{2}$ Institute of Scientific Instruments of the ASCR v.v.i., Kralovopolska, 147, 612 00, Brno, Czech Republic, bar@isibrno.cz

The article reports on certain artifacts that emerge during the in vitro diffusion-weighted imaging of physical samples. In this context, the authors analyze the influence of magnetic field inhomogeneity, temperature, or eddy currents and consider artifact mitigation procedures. A technique reducing the examined spurious effects was designed, experimentally verified, and denominated as the three measurement method. The technique proved to be useful mainly for the evaluation of a DWI image measured with a diffusion gradient in the $z$ axis, where the relative measurement error decreased to $3.38 \%$ (during measurement using two images, the relative error was greater than $19 \%$ ). For small errors within the measurement of diffusion constants of a deionized water sample $(<5 \%)$ it was necessary to select a $b$-factor value larger than $200 \cdot 10^{6} \mathrm{~s} \cdot \mathrm{m}^{-2}$. Temperature stabilization with accuracy better than $0.1{ }^{\circ} \mathrm{C}$ during the entire measuring process is a necessary prerequisite for the measurement of biological or material samples with relative accuracy lower than $1 \%$.
\end{abstract}

Keywords: Correction, diffusion, inhomogeneity, eddy currents, magnetic resonance

\section{INTRODUCTION}

$\mathrm{D}$ IFFUSION CONSTITUTES a random translational motion of molecules given by their thermal energy without requiring mass volume motion. This motion is described by the physical quantity diffusion coefficient $D$ [1]-[3]. During the diffusion time [4], the molecules may pass through regions having different diffusion coefficients $D$; thus, there occurs time dependence of the coefficient. For such cases, the term Apparent Diffusion Coefficient, $A D C$, was introduced (Le Bihan 1986 [5]); the term expresses the effective value of diffusion coefficient $D$ and is timeindependent. Diffusion is often an anisotropic quantity (the $D$-value differs depending on the direction), and therefore coefficient $D$ turns into tensor $\mathbf{D}$, which can be obtained only through measurement in six independent directions. The most commonly used method for the measurement of diffusion coefficient is the Pulsed Field Gradient Spin Echo (PFGSE) [6], a spin echo method [7], [8] with two diffusion gradients.

A significant source of errors may consist in the inaccuracy of parameters of generated gradient pulses. A non-negligible aspect affecting the accuracy of measurement of diffusion coefficients in biological tissues having short relaxation times $T_{1}$ and $T_{2}$ is brought by the parameters of the magnetic field gradient pulses; these parameters include the amplitude, the ramp time, and the length of pulse. To measure the time characteristics of magnetic field gradients, various MR methods are used; among these, the most interesting ones are the methods given in [9]-[17]. All of the techniques have been used to set precisely the pre-emphasis compensation in MR tomographs.

A multitude of articles such as [18]-[25] have dealt with the problem of image artifacts. The presented article contains a description of the Diffusion weighted imaging (DWI) and Diffusion tensor imaging (DTI) techniques as well as an analysis of the influence exerted by magnetic field inhomogeneity, eddy currents, and temperature on measurement errors. We designed the three measurement method to eliminate these spurious aspects. The effect of magnetic field deformation owing to magnetic susceptibility constitutes a significant element mainly in heterogeneous materials.

\section{METHODS OF MEASURING DIFFUSION COEFFICIENTS AND DIFFUSION TENSOR}

This chapter focuses on the problem of obtaining DWI and DTI images. Within the final subsection, the widely applied PFGSE method is analyzed with respect to parameters of the gradients that influence the accuracy of measurement.

\section{A. Diffusion Weighted Imaging (DWI)}

In DWI images, every image point (a pixel or a voxel) constitutes the value of diffusion coefficient $D$. This diffusion coefficient is calculated according to (1) [6].

$$
\frac{S}{S_{0}}=\mathrm{e}^{-b D}
$$

where quantity $S$ represents the image intensities measured with a gradient pulse and $S_{0}$ represents the intensities without the gradient pulse. In order to create a $2 \mathrm{D}$ image, we apply MR tomography to measure the 2D matrix of intensities $\mathbf{S}$ and the 2D matrix of intensities $\mathbf{S}_{\mathbf{0}}$. Using (1), we obtain a diffusion-weighted image for every image point of the matrix [26].

\section{B. Diffusion Tensor Imaging (DTI)}

Diffusion tensor imaging (DTI) is a comparatively new method of magnetic resonance-based medical examination; currently, this technique is the only one capable of visualizing close structural details of brain white matter or cardiac muscle fibers [25]-[28]. In DTI images, every voxel is represented by means of an ellipsoid. In order to visualize the ellipsoid, it is necessary to perform at least 7 measurements for different directions of the gradient and to 
evaluate the DWI images. One image will be without gradients $\left(\mathbf{S}_{\mathbf{0}}\right)$, and images $\mathbf{S}_{\mathbf{1}}$ to $\mathbf{S}_{\mathbf{6}}$ will be with gradients in different directions (for example, in axes $x, y, z, x y, y z$ and $x z$ ). Then, we can calculate the diffusion tensor according to the following relation and thus visualize one voxel in the DTI image.

$$
\frac{S}{S_{0}}=e^{-\mathbf{b} \cdot \mathbf{D} \cdot \mathbf{b}^{T}}
$$

where $\mathbf{b}$ is the vector containing information related to not only the gradient magnitude, but also to its orientation [29]. Matrix D constitutes a symmetrical tensor containing diffusion coefficients $D$ calculated from the measurement along six independent coordinate directions.

A voxel is visualized through the use of an ellipsoid. The drawing of the ellipsoid is only possible if we know the proper values $\lambda$ and proper vectors $\mathbf{v}$. These are obtained from tensor D by means of the "diagonalization" process, which is shown in

$$
\mathbf{D}=\left[\begin{array}{ccc}
D_{\mathrm{xx}} & D_{\mathrm{xy}} & D_{\mathrm{xz}} \\
D_{\mathrm{yx}} & D_{\mathrm{yy}} & D_{\mathrm{yz}} \\
D_{\mathrm{zx}} & D_{\mathrm{zy}} & D_{\mathrm{zz}}
\end{array}\right] \stackrel{\text { diagonalization }}{\longrightarrow} \lambda_{1}, \lambda_{2}, \lambda_{3}, \mathbf{v}_{1}, \mathbf{v}_{2}, \mathbf{v}_{3} .
$$

By modifying (3) it is possible to obtain the following equation, which can also be used for the calculation of individual diffusion coefficients [26]

$$
\mathbf{b} \cdot \mathbf{D} \cdot \mathbf{b}^{T}=\left[\sqrt{b_{\mathrm{x}}}, \sqrt{b_{\mathrm{y}}}, \sqrt{b_{\mathrm{z}}}\right] \cdot\left[\begin{array}{ccc}
D_{\mathrm{xx}} & D_{\mathrm{xy}} & D_{\mathrm{xz}} \\
D_{\mathrm{yx}} & D_{\mathrm{yy}} & D_{\mathrm{yz}} \\
D_{\mathrm{zx}} & D_{\mathrm{zy}} & D_{\mathrm{zz}}
\end{array}\right] \cdot\left[\begin{array}{c}
\sqrt{b_{\mathrm{x}}} \\
\sqrt{b_{\mathrm{y}}} \\
\sqrt{b_{\mathrm{z}}}
\end{array}\right] .
$$

\section{Methods of DWI and DTI data measurement}

Within the measurement of the diffusion coefficient, the most accurate results can be achieved using the PFGSE method. In this technique, two RF pulses are utilized; however, as the principle of the method is generally well known [23]-[25], we will now only describe the manner of achieving the highest possible degree of accuracy in DWI data measurement.

The coefficient $D$ depends on the temperature, the field homogeneity, and characteristics of the gradient pulses. For the description of time intervals and magnitude of gradient pulses, the $b$-factor quantity is introduced. Diffusion gradients $G$ do not always assume a rectangular shape. In certain valid cases, for example, a trapezoidal form or gradients showing a half-sinusoidal waveform can be used. One of the reasons for the selection of these gradient pulse shapes consists in a marked deceleration of the leading and trailing edges, the generation of smaller eddy currents in the surrounding conductive materials, and a significant reduction of the magnetic field gradient pulses. Yet, in spite of this, eddy currents induce artifacts in the measured diffusion images.

For the $b$-factor calculation of any diffusion gradient waveform, we designed the program in Matlab. This program utilizes the following equation:

$$
b=\gamma^{2} \cdot \int_{0}^{T_{\mathrm{E}}}\left[\int_{0}^{t} G\left(t^{\prime}\right) \mathrm{d} t^{\prime}\right]^{2} \mathrm{~d} t
$$

If the diffusion gradients are of a rectangular shape, it is possible to modify (5):

$$
b=\gamma^{2} G_{\mathrm{D}}^{2} \delta^{2}(\Delta-\delta / 3) .
$$

If the amplitudes (or time integrals) of the two gradients are not identical, there will be a systematic error $\delta_{\mathrm{D}}$ in the determination of diffusion coefficient $D$. The magnitude of this error, established by numerical calculation for the amplitude error of generated gradient $G_{1}$ (for constant $G_{2}$ ) or $G_{2}$ (for constant $G_{1}$ ), is given in Fig.1 for a simple measuring sequence.

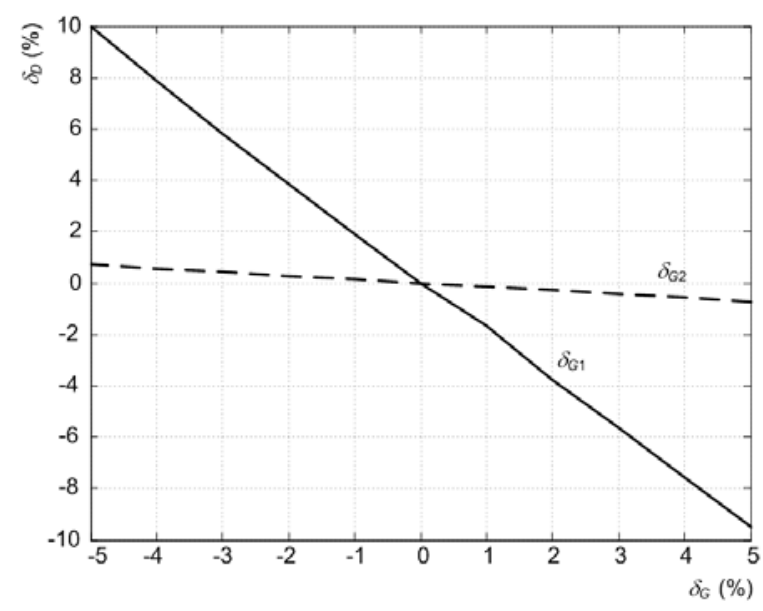

Fig.1. The systematic error of diffusion coefficient $\delta_{D}$ depending on the magnitude of amplitude errors $\delta_{\mathrm{G} 1}$ and $\delta_{\mathrm{G} 2}$ of generated gradients $G_{1}$ and $G_{2}$.

It follows from Fig.1 that error $\delta_{D}$ is significantly influenced by the error of amplitude $G_{1}$. The magnitude of this error does not differ from a situation when a change of both gradients in the pulse sequence occurs. In this case, the error can be expressed by

$$
\frac{\delta_{\mathrm{D}}}{D}=2 \frac{\delta_{\mathrm{G}}}{G}
$$

The accuracy of setting the time intervals in the pulse sequence is high and the systematic error of the pulse sequence can be neglected.

\section{ARTIFACTS AND THEIR ELIMINATION IN VISUALIZING DWI AND DTI IMAGES}

All the experimental measurements were carried out using the MR tomograph $\left(B_{0}=4.7 \mathrm{~T} / 70 \mathrm{~mm},{ }^{1} \mathrm{H} \sim 200 \mathrm{MHz}\right)$ available at the Institute of Scientific Instruments, Academy of Sciences of the Czech Republic (ISI ASCR). The overview and composition of the measured samples are provided in Table1. Nickel sulphate and $\mathrm{NaCl}$ were applied for shortening the relaxation times as well as the total measurement time ( $\left.T_{1}=T_{2}=130 \mathrm{~ms}\right)$. 
Table1. The measured isotropic samples.

\begin{tabular}{|l|l|}
\hline Samples & Composition \\
\hline Solution 1 & Deionized water \\
\hline Solution 2 & 1 liter water, 1.2 gram $\mathrm{NiSO}_{4}$ and 2.6 gram $\mathrm{NaCl}$ \\
\hline Solution 3 & 1 liter water, 4.8 gram $\mathrm{NiSO}_{4}$ and 2.6 gram $\mathrm{NaCl}$ \\
\hline
\end{tabular}

\section{A. The effect of temperature on DWI images}

The principle of diffusion consists in the thermal motion of molecules. The measured value of the diffusion-weighted image intensity is therefore markedly dependent on the temperature; the expression of this dependence can be found in the Einstein-Stokes equation [25]

$$
D=\frac{k T}{2 \pi \eta r_{\mathrm{s}}},
$$

where $k\left[\mathrm{JK}^{-1}\right]$ - the Boltzmann constant, $T[\mathrm{~K}]$ - absolute temperature of the sample, $\eta\left[\mathrm{kg} \cdot \mathrm{m}^{-1} \mathrm{~s}^{-1}\right]$ - viscosity of the sample, and $r_{\mathrm{s}}[\mathrm{m}]$ - the effective molecular diameter.

The temperature dependence of diffusion is also described by Le Bihan [30]. During the in vitro measurement, a precondition for the thermal stabilization of the sample was experimentally established to secure the highest possible degree of accuracy in the measurement of physical samples.

In order to determine the thermal dependence of diffusion coefficient in various substances, we applied all isotropic samples quoted in Table1. The measured solutions were placed in a spherical glass vessel having an external diameter of $40 \mathrm{~mm}$, and the measurement of DWI images was performed on the MR tomograph. The thermal system based on the regulation of temperature of air flowing through the operating area maintained the sample temperature within the range of $20.0^{\circ} \mathrm{C}-30.0^{\circ} \mathrm{C}$ with an accuracy of $0.1^{\circ} \mathrm{C}$. In Fig.2, the measured dependence of diffusion in selected samples on the temperature is shown.

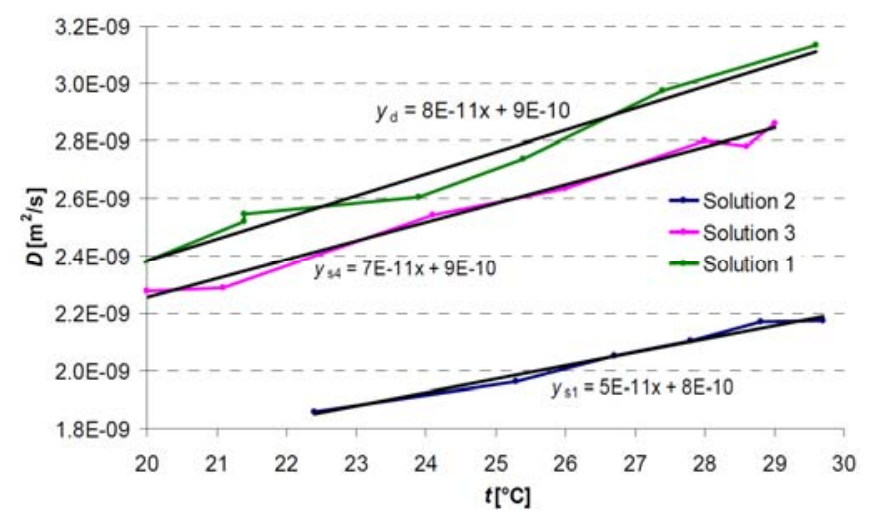

Fig.2. A diagram of the dependence of diffusion coefficient on the temperature for individual measured samples.

Solution 1 (deionized water) will change the diffusion coefficient, with a temperature variation of $1{ }^{\circ} \mathrm{C}$, by $5.10^{-11} \mathrm{~m}^{2} / \mathrm{s}$. Relative error of the diffusion coefficient in this sample of water is $2.17 \% / 1{ }^{\circ} \mathrm{C}$. In order to measure deionized water with relative error of $1 \%$, we have to stabilize the sample temperature with an accuracy of $0.5^{\circ} \mathrm{C}$.
For substances with slower diffusion rates, it is suitable to maintain the sample temperature with the accuracy rate of $0.1^{\circ} \mathrm{C}$.

\section{B. The effect of magnetic field inhomogeneity and eddy currents}

Magnetic susceptibility of the measured sample causes deformation of the stationary magnetic field [21]. This deformation is larger in heterogeneous materials. Inhomogeneity of the magnetic field at each pixel can be described using the magnetic field stationary gradient $G_{0}$, which is added to the stationary field within the field of the view area and to the diffusion gradients; the stationary gradient is effective through the entire duration of the experiment. Errors due to the presence of gradient $G_{0}$ are very large [25]. The effect of this field on the accuracy of the diffusion coefficient measurement should be minimized.

Large, rapidly switched magnetic field gradients induce eddy currents in electrically conductive structures of the MRI scanner, which in turn produce additional unwanted, rapidly and slowly decaying magnetic fields. In diffusion imaging, due to the limited gradient power and in order to achieve the desired $b$-factor, the gradients are applied for much longer; thus, the eddy currents do not tend to cancel themselves out. Furthermore, the most common form of sequence employed in the DT-MRI is the pulse-gradient spin echo with EPI readout [31], [32]. With such readout, the bandwidth in the phase-encoded direction is small; therefore any errors in achieving the desired gradient are exacerbated [25]. Eddy currents produce two basic spurious effects: 1 . The real gradient magnetic field in the measured sample differs from the required magnetic field, which leads to a difference between the real and the desired $b$-factor matrix. Thus, in the DWI and DTI, errors are generated in the diffusion coefficient magnitude; moreover, in DTI images there occurs erroneous drawing of the ellipsoid inclination. 2. Due to eddy currents, errors occur in the image intensity readout; thus, geometrical distortion of the DWI and DTI images is caused.

The method of eliminating eddy currents by means of bipolar diffusion gradients [32] can be applied in the measurement of faster diffusion. Postprocessing interpolation is described by Bodammer et. al [31]. If diffusion gradients of both polarities (described, for example, in [33]) are applied, it is possible to compensate the effect of stationary magnetic field inhomogeneities.

In order to improve the accuracy of diffusion coefficient measurement, we propose to measure three DWI images: without a gradient, with the positive diffusion gradient, and with the negative diffusion gradient. The diffusion coefficient-weighted image with the compensation of inhomogeneities and non-linearities of the magnetic field can be calculated from

$$
D=\frac{1}{b} \cdot \ln \left(\frac{S_{+} \cdot S_{-}}{S_{0}^{2}}\right),
$$

where $S_{0}$ is the measured signal intensity without the gradient pulse; $S_{+}$is the measured signal intensity with the 
positive diffusion gradient pulse; $\mathrm{S}$. is the measured signal intensity with the negative diffusion gradient pulse.

The magnitude of relative errors for the proposed three measurement method was experimentally verified by means of measuring the diffusion coefficient of deionized water (Solution 1) at the constant temperature of $20^{\circ} \mathrm{C}$; also, a comparison with the results of two measurements was performed (the diffusion gradient of only one polarity).

The primary step for us was to find the $b$-factor value for which the relative measurement errors are minimal; therefore, we measured the diffusion coefficient-weighted images for the $b$ within the range of 50 to $320 \mathrm{~s} . \mathrm{mm}^{-2}$. It is obvious from Fig.3 that the effects of magnetic field $\Delta B$ inhomogeneity are stronger for the $b$-factor of a small value; here, the reason consists in the use of a diffusion gradient having a small ratio of $G / \Delta B$. For the measurement of diffusion constants of the measured sample of deionized water with relative error smaller than $5 \%$, it is necessary to select a $b$-factor value larger than $200 \cdot 10^{6} \mathrm{~s} . \mathrm{m}^{-2}$.

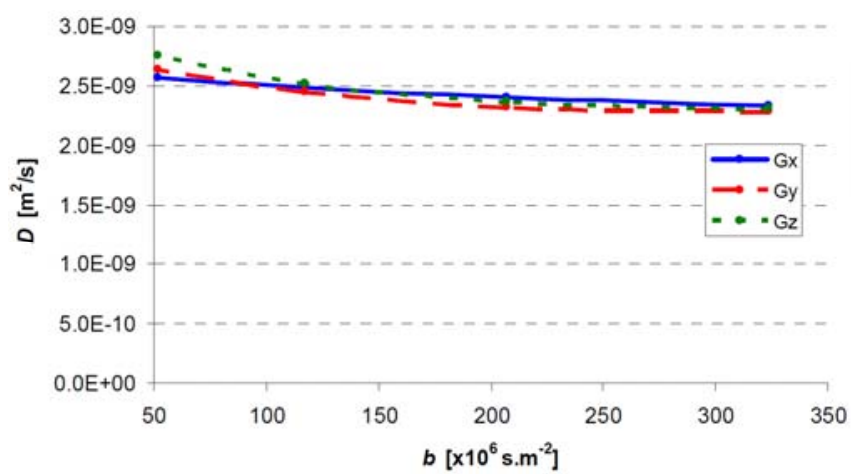

Fig.3. The dependence $D$ of deionized water on the $b$-factor magnitude in directions $x$, y and $z$ at the temperature of $20.0^{\circ} \mathrm{C}$.

In order to verify the three measurement method, we set the diffusion gradients in such a manner that the $b$-factor magnitude was $b=250 \cdot 10^{6} \mathrm{~s} . \mathrm{m}^{-2}$. The reference value was the diffusion constant of deionized water for $20.0^{\circ} \mathrm{C}$, which has the magnitude of $D=2.369 \cdot 10^{-9} \mathrm{~m}^{2} / \mathrm{s}$.

We obtained three images by means of the PFGSE measuring method, (Fig.4). Two images were measured with the diffusion gradients of the positive and negative polarities in the $x, y$ and $z$ axes. The third image measured was without a gradient. The DWI image calculation was performed using the algorithm quoted in Fig.5. By introducing individual intensities from the measured images in (9), we obtain the resulting DWI image.
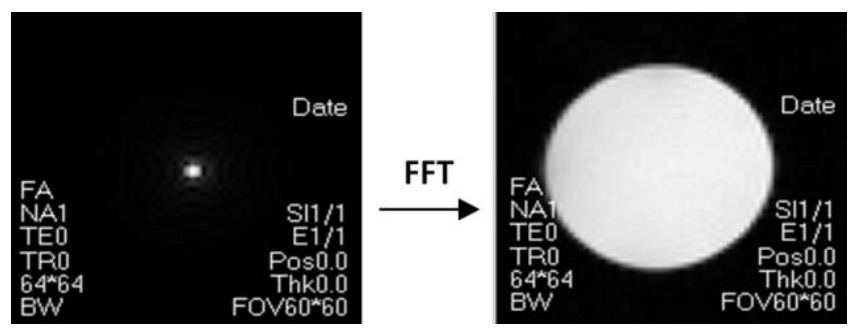

Fig.4. The measured image of deionized water without diffusion gradients. Left: the $k$-region; right: the image region.

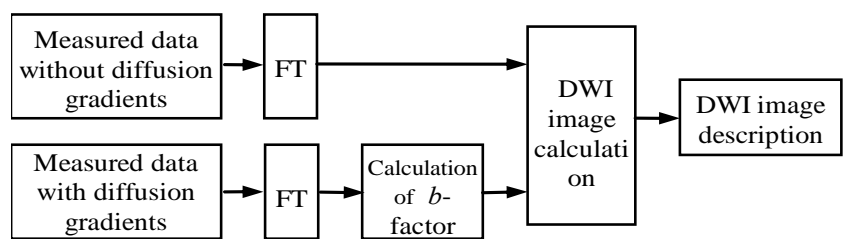

Fig.5. The algorithm for DWI image processing.

In order to obtain the diffusion coefficient from the processed DWI images, we selected the entire region representing deionized water in the image (a circle); from this region, the diffusion coefficient was calculated as a median of all the selected values.

Table2 (left) presents diffusion coefficients measured in the following order: a) from two images according to (1), namely from the zero-gradient and the positive-gradient images b) from the zero-gradient and the negative-gradient images c) from three images according to (9). The measurements were performed for all basic coordinate directions. The results in Table2 indicate marked influence of the stationary magnetic field inhomogeneity on relative error $\delta_{\mathrm{D}}$, which (for the diffusion coefficient measurement) is from two images, $\mathbf{S}_{\mathbf{z}^{+}}$and $\mathbf{S}_{\mathbf{z}-}, 28.12 \%$ and $-19.09 \%$. Using the three measurement method, we reduced the error to the magnitude of $4.36 \%$. Even though relative errors of the diffusion coefficient in directions $x$ and $y$ are smaller, they are reduced by the three measurement method.

One of the techniques of obtaining the DTI image consists in setting up the following combination of gradients $G$ :

$$
\begin{gathered}
{[0,0,0],[1,0,0],[0,1,0],[1,0,1],} \\
{\left[\frac{1}{\sqrt{2}}, \frac{1}{\sqrt{2}}, 0\right],\left[\frac{1}{\sqrt{2}}, 0, \frac{1}{\sqrt{2}}\right],\left[0, \frac{1}{\sqrt{2}}, \frac{1}{\sqrt{2}}\right] .}
\end{gathered}
$$

The magnitude of the gradients is set in such a manner that $b_{\mathrm{x}}=b_{\mathrm{y}}=b_{\mathrm{z}}=\sqrt{b_{\mathrm{x}}} \cdot \sqrt{b_{\mathrm{y}}}=\sqrt{b_{\mathrm{x}}} \cdot \sqrt{b_{\mathrm{z}}}=\sqrt{b_{\mathrm{y}}} \cdot \sqrt{b_{\mathrm{z}}}=221.5 \mathrm{~mm}^{2} / \mathrm{s}$. Thus, the calculation of diffusion coefficients $D$ is simplified. Table2 (right) summarizes $D$ measured in selected axes $(x y, y z, x z)$ of the coordinate systems for the positive and negative magnitudes of the diffusion gradients. The presented results show that the influence of stationary field inhomogeneity on the relative error of diffusion coefficient $\delta_{\mathrm{D}}$ is similar as in the main directions of the coordinate system. The error is significantly reduced by the three measurement method.

Table2. Left: Diffusion coefficients in the main coordinate directions. Right: Diffusion coefficients in various axes.

\begin{tabular}{|c|c|c|}
\hline Image & $D\left[\mathrm{~m}^{2} / \mathrm{s}\right]$ & $\delta_{\mathrm{D}}[\%]$ \\
\hline $\mathbf{S}_{\mathbf{x}^{+}}$ & $2.49 \mathrm{E}-09$ & 5.11 \\
\hline $\mathbf{S}_{\mathbf{x}^{-}}$ & $2.32 \mathrm{E}-09$ & -1.93 \\
\hline $\mathbf{S}_{\mathbf{x}}$ & $2.40 \mathrm{E}-09$ & 1.52 \\
\hline $\mathbf{S}_{\mathbf{z}^{+}}$ & $3.04 \mathrm{E}-09$ & 28.12 \\
\hline $\mathbf{S z}^{-}$ & $1.92 \mathrm{E}-09$ & -19.09 \\
\hline $\mathbf{S}_{\mathbf{z}}$ & $2.47 \mathrm{E}-09$ & 4.36 \\
\hline $\mathbf{S}_{\mathbf{y}^{+}}$ & $2.37 \mathrm{E}-09$ & -0.02 \\
\hline $\mathbf{S}_{\mathbf{y}-}$ & $2.22 \mathrm{E}-09$ & -6.21 \\
\hline $\mathbf{S}_{\mathbf{y}}$ & $2.29 \mathrm{E}-09$ & -3.34 \\
\hline
\end{tabular}

\begin{tabular}{|c|c|c|}
\hline Image & $D\left[\mathrm{~m}^{2} / \mathrm{s}\right]$ & $\delta_{\mathrm{D}}[\%]$ \\
\hline $\mathbf{S}_{\mathrm{xy}+}$ & $2.40 \mathrm{E}-09$ & 1.24 \\
\hline $\mathbf{S}_{\mathrm{xy}-}$ & $2.14 \mathrm{E}-09$ & -9.46 \\
\hline $\mathbf{S}_{\mathrm{xy}}$ & $2.27 \mathrm{E}-09$ & -4.34 \\
\hline $\mathbf{S}_{\mathrm{xz}+}$ & $2.60 \mathrm{E}-09$ & 9.75 \\
\hline $\mathbf{S}_{\mathrm{xz}-}$ & $1.99 \mathrm{E}-09$ & -15.88 \\
\hline $\mathbf{S}_{\mathrm{xz}}$ & $2.29 \mathrm{E}-09$ & -3.12 \\
\hline $\mathbf{S}_{\mathrm{yz}+}$ & $2.94 \mathrm{E}-09$ & 23.98 \\
\hline $\mathbf{S}_{\mathrm{yz}}-$ & $1.95 \mathrm{E}-09$ & -17.50 \\
\hline $\mathbf{S}_{\mathrm{yz}}$ & $2.45 \mathrm{E}-09$ & 3.38 \\
\hline
\end{tabular}


Images weighted by coefficients $D_{\mathrm{xx}}, D_{\mathrm{yy}}, D_{\mathrm{zz}}$ were introduced in the diffusion tensor matrix (see (4) and (9)). The measurement of images weighted by diffusion coefficients in $x y, x z$ and $y z$ axes provided coefficients $D_{\mathrm{xy}}^{\prime}$, $D_{\mathrm{xz}}^{\prime}$ a $D_{\mathrm{yz}}^{\prime}$. Before being introduced into the diffusion tensor matrix, these coefficients must be recalculated according to

$$
\begin{aligned}
& D_{\mathrm{xy}}=D_{\mathrm{xy}}^{\prime}-0.5 \cdot D_{\mathrm{xx}}-0.5 \cdot D_{\mathrm{yy}} \cdot \\
& D_{\mathrm{xz}}=D_{\mathrm{xz}}^{\prime}-0.5 \cdot D_{\mathrm{xx}}-0.5 \cdot D_{\mathrm{zz}} . \\
& D_{\mathrm{yz}}=D_{\mathrm{yz}}^{\prime}-0.5 \cdot D_{\mathrm{yz}}-0.5 \cdot D_{\mathrm{yz}} .
\end{aligned}
$$

After the introduction of individual diffusion coefficients $D$ in (2), we get the resulting diffusion tensor $\mathbf{D}$. This tensor represents one image point (voxel), and in the image it will be geometrically drawn as an ellipsoid. The ellipsoid carries information concerning both the magnitude of diffusion in individual coordinate directions and the direction of the diffusion propagation. Therefore, the DTI method can be applied for the imaging of muscle and neural fibers in the human body [25]-[28], [34]. As we measured a DTI image having the size of $64 \times 64$ voxels, the total of 4096 ellipsoids was calculated. In Fig.6 (left), the DTI image of the measured deionized water is drawn; in the same figure (right), a voxel representing the ellipsoid is selected.
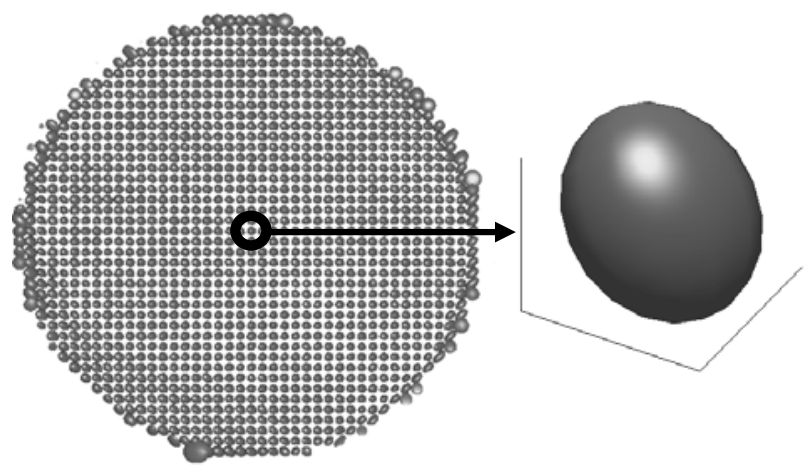

Fig.6. The resulting DTI image of deionized water and the selected ellipsoid (magnified).

Deionized water can be regarded as an isotropic environment, and therefore the tensor should be expressed in the ideal case - by a sphere. It is shown in Fig. 6 that the result we obtain is not a sphere, but rather a slightly turned ellipsoid. Here, even though we used the three measurement method, the total compensation of all artifacts does not occur (Table2). Fig.7 (left) presents the results of our measurement of an isotropic sample - saline solution. The right section of the figure shows the results of our measurement of an anisotropic sample - cabbage.

The images a) and d) were obtained from the calculation of the three measurement method (9); the DTI images b) and e) were calculated using (3) and (4); the images c) and d) show a sample of one ellipsoid (the size of the ellipsoid is determined by their eigenvalues $\lambda$, while the inclination of the ellipsoid is determined by eigenvectors v). By using (9) we were able to eliminate inhomogeneities and eddy currents, which is shown in Fig.7c). In Fig.7f), not all the ellipsoids are of the same size as in the case of isotropic material; the ellipsoids exhibit different sizes and inclinations according to the growth of fibers within the sample of cabbage.

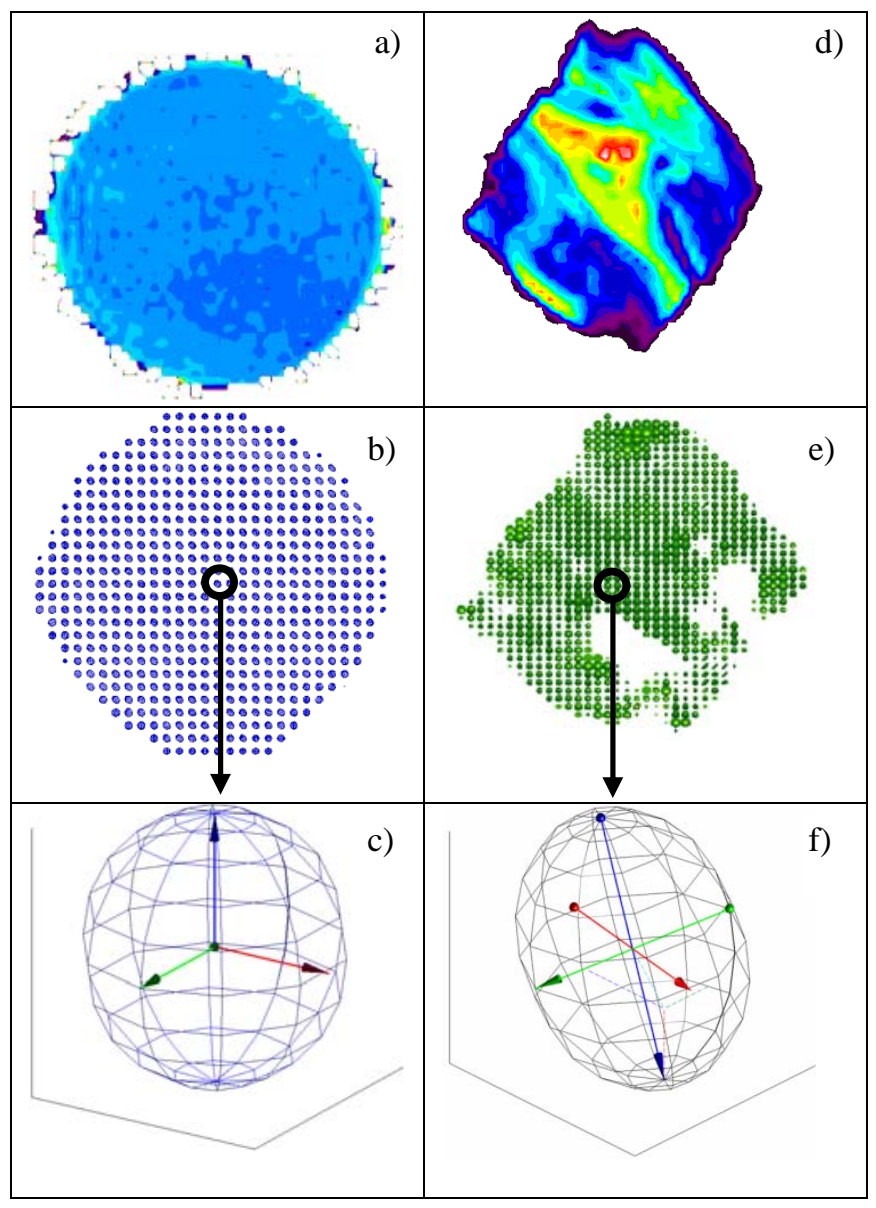

Fig.7. Left: images of an isotropic sample - saline solution; right: images of anisotropic sample - a piece of cabbage (DWI a),d); DTI b),e); a selected sample of ellipsoid whose direction is determined by its eigenvectors and the size of its eigenvalues c), f)).

\section{The b-factor influence on the diffusion weighted image}

According to (1), it is possible to calculate the diffusion coefficient by means of approximating the decrease in the magnitude of spin echoes measured for different values of the $b$-factor. This procedure of the measurement and calculation of $D$ can be advantageously used for homogeneous materials with constant diffusion. The procedure is also well applicable for heterogeneous materials having several regions with different diffusion, mainly because it allows us to perform the approximation of behavior of the spin echoes' magnitude decrease for two or more exponential functions.

The $b$-factor magnitude is given by the characteristics of diffusion gradient pulses and has a significant influence on the signal/noise ratio in the image [6], [8]. An increase of the $b$-factor can be achieved by means of increasing the magnitude and length of the diffusion gradient and through protracting the diffusion time. The increasing of the gradients' magnitude is limited by the applied instrument 
(tomograph) and expands the influence of eddy currents on the accuracy of the measurement as well as on the generation of artifacts in the image. Protraction of both the gradients' length and the diffusion time significantly decreases the signal/noise ratio, mainly for small values of $D$. Therefore, it is necessary to find optimal parameters of the diffusion gradients.

For the experimental verification of relative error magnitudes in the measurement of diffusion constants, the images were measured for five $b$-factor magnitudes realized through a change of the diffusion gradient magnitudes: $b=(0,51.8,116.7,207.4,324.1) \mathrm{s} . \mathrm{m}^{-2}$. The measurement was performed individually for both the positive and the negative diffusion gradients. In order to eliminate the influence of magnetic field inhomogeneities on the measured diffusion coefficient, it is suitable to average the results of both measurements (similarly as in the three measurement method).

By means of approximating the measured relations between intensities with and without gradient $S / S_{0}$ (according to (1)) from the selected image region, we obtain the diffusion weighted image. Fig. 8 shows the resulting diffusion coefficients determined by the approximation from two to five measured images with diffusion gradients.

Images measured with positive gradients are evaluated individually, and such individual evaluation applies also to images measured with negative gradients. The meaning of numbers on the $x$-axis is as follows:

5 - measured at $b=(0,51.8,116.7,207.4,324.1) \mathrm{s.m}^{-2}$;

4 - measured at $b=(51.8,116.7,207.4,324.1){\mathrm{s} . \mathrm{m}^{-2}}^{2}$;

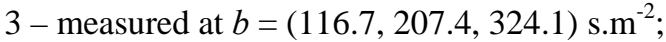

2 - measured at $b=(207.4,324.1) \mathrm{s} . \mathrm{m}^{-2}$.

According to the diagram, it is sufficient to perform the measurement with gradient magnitudes of $b=(116.7,207.4$, 324.1) $\mathrm{s.m}^{-2}$ and, subsequently, to approximate the obtained images (fitting) using exponential equation (1).

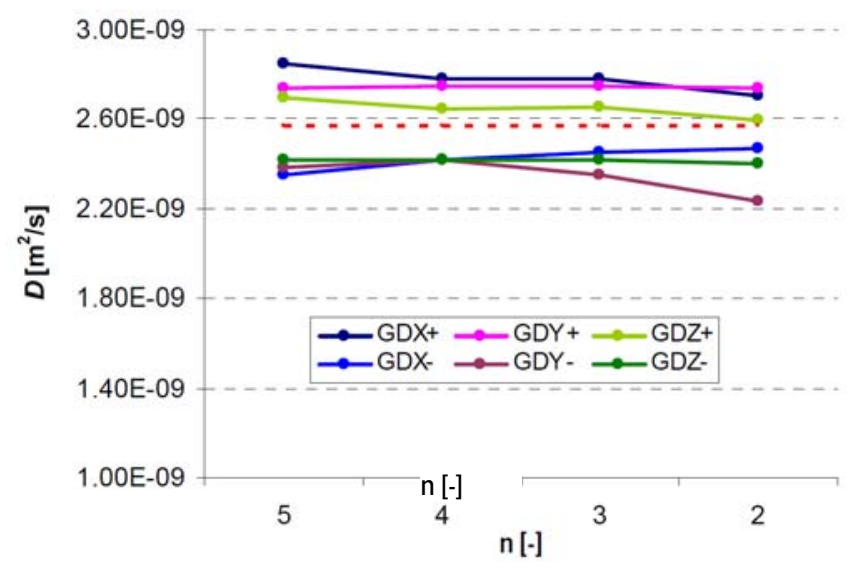

Fig.8. A diagram of the evaluation of diffusion from approximated images obtained at different values of $b$ (both the positive and the negative polarities are evaluated individually), temperature of $22.6^{\circ} \mathrm{C}$.

By averaging the data measured with positive and negative diffusion gradients, we obtain very accurate values of the measured deionized water diffusion coefficient. The known value of the diffusion coefficient at the temperature of $22.6^{\circ} \mathrm{C}$ is $D=2.566 .10^{-9} \mathrm{~m} . \mathrm{s}^{-2}$. Table3 contains an overview of diffusion coefficients obtained from the measured data with positive and negative gradients in individual axes and presents a quotation of average values of the coefficients, which provides us with sufficiently accurate results. The results in the table correspond to the approximation of 5 measured images with different values of the diffusion gradient.

Table3. The diffusion coefficient obtained through approximation of the measured images at different magnitudes of the $b$-factor.

\begin{tabular}{|c|c|c|}
\hline Image & $D\left[\mathrm{~m}^{2} / \mathrm{s}\right]$ & $\delta_{\mathrm{D}}[\%]$ \\
\hline $\mathbf{S}_{\mathrm{x}+}$ & $2.846 \mathrm{E}-09$ & 10.93 \\
\hline $\mathbf{S}_{\mathrm{x}-}$ & $2.346 \mathrm{E}-09$ & -8.56 \\
\hline $\mathbf{S}_{\mathrm{x} \phi}$ & $2.596 \mathrm{E}-09$ & 1.18 \\
\hline $\mathbf{S}_{\mathrm{y+}}$ & $2.742 \mathrm{E}-09$ & 6.87 \\
\hline $\mathbf{S}_{\mathrm{y}-}$ & $2.384 \mathrm{E}-09$ & -7.08 \\
\hline $\mathbf{S}_{\mathrm{y} \phi}$ & $2.563 \mathrm{E}-09$ & -0.10 \\
\hline $\mathbf{S}_{\mathrm{z}+}$ & $2.695 \mathrm{E}-09$ & 5.04 \\
\hline $\mathbf{S}_{z^{-}}$ & $2.421 \mathrm{E}-09$ & -5.64 \\
\hline $\mathbf{S}_{\mathrm{z} \phi}$ & $2.558 \mathrm{E}-09$ & -0.30 \\
\hline
\end{tabular}

\section{DISCUSSION AND CONCLUSION}

In spite of the fact that a large number of applications enable us to utilize relative changes of diffusion coefficients, exact determination of the diffusion coefficient in homogeneous and heterogeneous materials is regarded as a very important step. The accuracy of the measurement method is even more significant in the area of DTI images.

With respect to a highly accurate generation of the time and shape parameters of magnetic field gradients in current MR tomographs, relative errors $\delta_{\delta}, \delta_{\Delta}$ a $\delta_{\mathrm{G}}$ can be considered neglectable. However, changes of the sample temperature during the measurement constitute an essential problem. From temperature dependencies of the samples measured in vitro there follows the requirement of exact stabilization of the sample temperature (with the accuracy of $\left.0.1^{\circ} \mathrm{C}\right)$.

Magnetic field inhomogeneity is effective through the entire duration of the experiment and, according to the diffusion gradient polarity, it causes the increase or decrease of the gradient strength. This change constitutes the reason for large relative errors of the measured diffusion coefficient; these errors range within tens of percent. Despite its very good homogeneity, the tomograph stationary field is deformed by magnetic susceptibility of the sample. In the case of heterogeneous samples, the magnetic field deformations are not eliminable.

In order to facilitate the elimination of magnetic field inhomogeneities, we designed and experimentally verified the three measurement method. This technique is based on applying the PFGSE procedure to measure one image without a diffusion gradient, another image with a positive diffusion gradient in a coordinate direction, and yet another image in the same coordinate direction but with reverse polarity of the gradient. The described method proved to be very useful mainly for the evaluation of a DWI image measured with the diffusion gradient in the $z$ axis, where the relative measurement error decreased to $3.38 \%$ (during the measurement of two images, the relative error was greater than $19 \%)$. While it is true that the diffusion coefficient 
calculation from two images is less time-consuming than the proposed method utilizing three measurements, it also has to be noted that such type of calculation provides a lower degree of accuracy.

The measurement and approximation of the spin echoes' decrease for several values of $b$-factors is equally advantageous as the above-quoted method. If we apply diffusion gradients of only one polarity, the relative measurement errors are large. However, the use of gradients having both polarities and the averaging of the resulting values of the diffusion coefficient markedly help to decrease the measurement error; the error was lower than $1.2 \%$ for all coordinate directions within the experiment carried out using the ISI Brno tomograph. The technique of approximation of the measured images with different $b$ factors is even more advantageous than the three measurement method (see Table2, Table3); nevertheless, this procedure involves a substantial drawback consisting in the much greater amount of time required.

In comparison with the DWI technique, the DTI image evaluation method applicable for the measurement of heterogeneous materials exhibits a substantially higher degree of sensitivity to magnetic field inhomogeneities and eddy currents, mainly because the artifacts exert an influence not only on the size of the diffusion ellipsoid in individual coordinate directions, but also on its tilt (compare relations (10), (11), (12)). This characteristic may cause problems during the tractographic reconstruction of fibers (muscular or neural) [35], [36]. Through the use of the three measurement method or by measuring for several $b$-factors, this error can be very well corrected.

\section{ACKNOWLEDGMENT}

The research described in the paper was financially supported by the project of European Commission and Ministry of Education, Youth and Sports of the Czech Republic (project No. CZ.1.05/2.1.00/01.0017), by the research grant GACR 102/11/0318, GACR 102/12/1104, and grant no. FEKT-S-11-5/1012.

\section{REFERENCES}

[1] Fick, A. (1855). Concerns diffusion and concentration gradient. Annalen der Physik und Chemie, 170, 59.

[2] Fick, A. (1855). Über Diffusion. Annalen der Physik und Chemie, 94, 59-86.

[3] Brown, R. (1828). On the general existence of active molecules in organic and inorganic bodies. Philosophical Magazine, 4, 161-173.

[4] Einstein, A. (1905). Über die von der molekularkinetischen Teorie der Wärme geforderte Bewegung von in ruhenden Flüssigkeiten suspendierten Teilchen. Annalen der Physik und Chemie, 17, 549-560.

[5] Bihan, D., et al. (1986). MR imaging of intravoxel incoherent motions: Application to diffusion and perfusion in neurologic disorders. Radiology, 161 (2), 401-407.

[6] Stejskal, E.O., Tanner, J.E. (1965). Spin diffusion measurements: Spin echoes in the presence of a time- dependent field gradient. Journal of Chemical Physics, 42 (1), 288-292.

[7] Frollo, I., Andris, P., Pribil, J., Vojtisek, L., Dermek, T., Valkovic, L. (2010). Measurement and imaging of planar electromagnetic phantoms based on NMR imaging methods. Measurement Science Review, 10 (3), 97-101.

[8] Juras, V., Szomolanyi, P., Gäbler, S., Frollo, I., Tratting, S. (2009). The relationship between MR parameters and biomechanical quantities of loaded human articular cartilage in osteoarthritis: An in-vivo study. Measurement Science Review, 9 (5), 127-130.

[9] Tanner, J.E. (1970). Use of the stimulated echo in NMR diffusion studies. Journal of Chemical Physics, 52 (5), 2523-2526.

[10] Senaj, V., Guillot, G., Darrasse, L. (1998). Inductive measurement of magnetic field gradients for magnetic resonance imaging. Review of Scientific Instruments, 69 (6), 2400-2405.

[11] Robertson, S., Hughes, D.G., Liu, Q., Allen, P.S. (1992). Analysis of the temporal and spatial dependence of eddy current fields in a $40 \mathrm{~cm}$ bore magnet. Magnetic Resonance in Medicine, 25 (1), 158-166.

[12] Qysong, R.E., Lowe, I.J. (1993). A simple method of measuring gradient induced eddy currents to set compensation networks. Magnetic Resonance in Medicine, 29 (1), 119-121.

[13] Balcom, B.J., Bogdan, M., Armstrong, R.L. (1996). Single-point imaging of gradient rise, stabilization, and decay. Journal of Magnetic Resonance, 118 (1), 122-125.

[14] Jellus, V., Sharp, J.C., Tomanek, B., Latta, P. (2003). An NMR technique for measurement of magnetic field gradient waveforms. Journal of Magnetic Resonance, 162 (1), 189-197.

[15] Chaabane, L., Favre, B., Desgoutte, P., Deguin, A., Lapray, C., Briguet, A. (1997). A multiprobe magnetometer for analysis of local field distortions induced by pulsed gradients. Measurement Science \& Technology, 8 (3), 332-337.

[16] Bartusek, K., Puczok, V. (1993). An NMR multifid method for measurement of magnetic field gradient. Measurement Science \& Technology, 4 (3), 357-361.

[17] Bartusek, K., Gescheidtova, E. (2002). Instantaneous frequency of spin echo method for gradient magnetic fields measurement in MR systems. Journal of Electrical Engineering, 53 (10), 49-52.

[18] Jones, D.K., Cercignani, M. (2010). Twenty-five pitfalls in the analysis of diffusion MRI data. NMR in Biomedicine, 23 (7), 803-820.

[19] Valkovic, L., Windischberger, C. (2010). Method for geometric distortion correction in fMRI based on three echo planar phase images. Measurement Science Review, 10 (4), 116-119.

[20] Pribil, J., Horacek, J., Horak, P. (2011). Two methods of mechanical noise reduction of recorded speech during phonation in an MRI device. Measurement Science Review, 11 (3), 92-98. 
[21] Marcon, P., Bartusek, K., Burdkova, M., Dokoupil, Z. (2011). Magnetic susceptibility measurement using 2D magnetic resonance imaging. Measurement Science and Technology, 22 (10), art. no. 105702.

[22] Walker, L., Chang, L.C., Koay, C.G., Sharma, N., Cohen, L., Verma, R., Pierpaoli, C. (2011). Effects of physiological noise in population analysis of diffusion tensor MRI data. Neuroimage, 54 (2), 1168-1177.

[23] Bartusek, K., Gescheidtova, E. (2006). Testing the quality of magnetic gradient fields for studying selfdiffusion processes by magnetic resonance methods. Measurement Science \& Technology, 17 (8), 2256-2262.

[24] Bartusek, K., Gescheidtova, E. (2008). MRI method of diffusion measurement in heterogeneous materials. Measurement Science \& Technology, 19 (4), art. no. 045504 .

[25] Johansen-Berg, H., Behrens, T.E.J. (2009). Diffusion MRI: From Quantitative Measurement to in Vivo Neuroanatomy. Elsevier.

[26] Susumu, M. (2009). Introduction to Diffusion Tensor Imaging. Elsevier.

[27] Kerkovsky, A., Sprlakova-Pukova, A., et. al. (2010). Diffusion tensor imaging - soucasne moznosti MR zobrazeni bile hmoty mozku. Ceska a slovenska neurologie a neurochirurgie, 73 (106), 136-142.

[28] Tournier, J.D., Mori, S., Leemans A. (2011). Diffusion tensor imaging and beyond. Magnetic Resonance in Medicine, 65 (6), 1532-1556.

[29] Leemans, A., Jones, D.K. (2009). The $b$-matrix must be rotated when correcting for subject motion in DTI data. Magnetic Resonance in Medicine, 61 (6), 1336-1349.
[30] Le Bihan, D., Mattiello, J., Levin, R.L. (1995). Noninvasive temperature imaging by MRI: A review. Biomedical Thermology, 13, 25-41.

[31] Bodammer, N., Kaufmann, J., Kanowski, M., Tempelman., C. (2004). Eddy current correction in diffusion-weighted imaging using pairs of images acquired with opposite diffusion gradient polarity. Magnetic Resonance in Medicine, 51 (1), 188-193.

[32] Alexander, A.L., Tsuruda, J.S., Parker, D.L. (1997). Elimination of eddy current artifacts in diffusionweighted echo-planar images: The use of bipolar gradients. Magnetic Resonance Medicine, 38, 1016-1021.

[33] Bartusek, K., Gescheidtova, E., Mikulka, J. (2010). Data processing in studying biological tissues, using MR imaging techniques. In 33th International Conference on Telecommunications and Signal Processing (TSP 2010), 17-20 August 2010. Budapest: Asszisztencia Szervezö Kft., 171-175.

[34] Mikulka, J., Gescheidtova, E., Bartusek, K. (2012). Soft-tissues image processing: Comparison of traditional segmentation methods with 2D active contour methods. Measurement Science Review, 12 (4), 153-161.

[35] Irfanoglu M.O., Walker, L. Sarlls, J., Marenco, S., Pierpaoli, C. (2012). Effects of image distortions originating from susceptibility variations and concomitant fields on diffusion MRI tractography results. Neuroimage, 61 (1), 275-288.

[36] Mikulka, J. (2011). ImageJ plug-ins for microscopic image processing. In 34th International Conference on Telecommunications and Signal Processing (TSP 2011), 18-20 August 2011. IEEE, 541-543.

Received April 25, 2012. Accepted September 17, 2012. 